\title{
Intriguing Mixed Pathologic Features in a Case of Dementia With Lewy Bodies
}

Brian J. Balin, PhD; Christine J. Hammond, MS; Katherine E. Galluzzi, DO

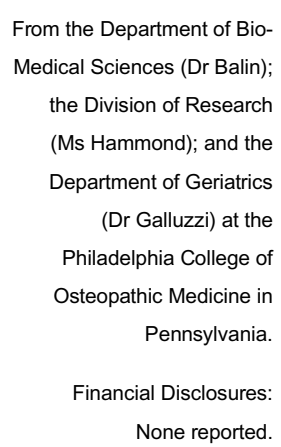

From the Department of BioMedical Sciences (Dr Balin); the Division of Research (Ms Hammond); and the Department of Geriatrics (Dr Galluzzi) at the Philadelphia College of Osteopathic Medicine in Pennsylvania.

Financial Disclosures: None reported.

Support: The Stanley E. Essl, DO '75 Endowed Memorial

Research Fund for Neurodegenerative Diseases and the Center for Chronic Disorders of Aging at PCOM provided funding for this study.

Address correspondence to

Brian J. Balin, PhD,

Department of Bio-Medical Sciences, Philadelphia College of Osteopathic Medicine, 4170 City Ave, 338 Evans Hall, Philadelphia,

PA 19131-1610.

Email: brianba@pcom.edu

Submitted

December 6, 2018; revision received January 7, 2019; accepted

February 19, 2019.
Neuropathologic confirmation of dementia with Lewy bodies (DLB) involves labeling cytoplasmic Lewy body inclusions for $\alpha$-synuclein in cortical and subcortical neurons. The authors studied the postmortem brain of a 78-year-old man who had a diagnosis of DLB by exclusion. The patient had symptoms ascribed to DLB that included fluctuating cognitive changes in attention and executive function with progression to dementia, visual hallucinations, and parkinsonism. Sections from the olfactory bulbs and cortical and subcortical regions were stained with periodic acid-Schiff, as well as immunolabeled with antibodies specific for $\alpha$-synuclein, tau protein, $\beta$-amyloid 1-42, and Chlamydia pneumoniae. Most regions demonstrated mixed neuropathologic features, and $\alpha$-synuclein was notable in Lewy bodies in the amygdala and hippocampus. Periodic acid-Schiff-positive staining was noted in bodies in the amygdala and olfactory bulbs. In this case of DLB, neuropathologic inclusions were consistent with the disease diagnosis, but also with Alzheimer disease and other neurodegenerative diseases, such as polyglucosan body disease.

J Am Osteopath Assoc. 2019;119(9):632-636

doi:10.7556/jaoa.2019.106

Keywords: $\alpha$-synuclein, Alzheimer disease, dementia, Lewy bodies

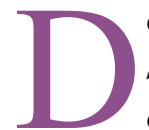
ementia with Lewy bodies (DLB), now classified in the Diagnostic and Statistical Manual of Mental Disorders, Fifth Edition ${ }^{1}$ as neurocognitive disorder with Lewy bodies, is the second most common neurodegenerative condition behind Alzheimer disease (AD). ${ }^{2}$ Typical age of onset of DLB ranges from 70 to 85 years, and unlike $\mathrm{AD}$, it is more common in men than in women. ${ }^{3}$ Clinical and diagnostic features typically include visual hallucinations, deficits in attention and alertness that inexorably progress to dementia, and motor abnormalities (parkinsonian features) that follow the initial cognitive impairment. ${ }^{4}$ Closely related conditions are Parkinson disease (PD) and PD dementia (PDD). Parkinson disease is principally characterized as a movement disorder with bradykinesia, rigidity, tremor at rest, and gait instability with response to levodopa therapy, ${ }^{5}$ and PDD is characterized when cognitive impairment develops a year or more after the appearance of motor abnormalities (parkinsonism). ${ }^{6}$ Dementia with Lewy bodies is clinically distinguishable from PDD because the cognitive impairment manifests well before any parkinsonian symptoms. Lewy bodies and Lewy neurites consisting of $\alpha$-synuclein are present in DLB, often in conjunction with amyloid plaques and tau tangles characteristic of $\mathrm{AD} .^{7}$ Brain regions commonly involved in DLB are neocortical, limbic, and brainstem, and the levels of involvement of each can influence the variation of symptoms from case to case. ${ }^{8}$ 


\section{Report of Case}

A 74-year-old man was referred to a neurologist by his primary care physician with symptoms including frequent falls, recurrent visual hallucinations, loss of smell and taste, and panic attacks. By exclusion, the neurologist diagnosed DLB. For the next 4 years, his symptoms progressed to include gait and vision problems, increasing cognitive deficits, speech and emotional issues, and changes in sensory input. Motor changes were characterized by festinating gait, ataxia, and loss of balance. The patient's ability to read was lost, and he had frequent visual hallucinations accompanied by loss of concentration, word-finding difficulty, and agnosia. Interestingly, he could differentiate musical instruments. The patient had difficulty with verbal expression and eventually adopted a blank stare. His family reported that he could "cry at the drop of a hat" and occasionally emoted angrily. The patient had sensory problems, and he could not distinguish between hot and cold. Near the end of his life, the patient developed a heightened startle response, was unable to easily breathe through the nose, and had worsening dysphagia. Three months before his death at the age of 78 years, the patient was nonambulatory, had taken in very little nutrition, and had become increasingly withdrawn. The death certificate listed "Advanced Lewy Body Dementia" as the cause of death. Per an advance discussion and consent by the family, the postmortem brain was removed for analysis.

\section{Medical History}

The patient's comorbid conditions included hypertension and coronary artery disease after cardiac stents and permanent pacemaker placement. He had undergone chemotherapy for basal cell carcinoma and had a history of renal calculi and varicella zoster virus infection (shingles). The patient was a lifelong smoker and may have had transient ischemic attacks but without diagnosed stroke or hydrocephalus. Early history included physical trauma from a fall, a car accident with resultant vertebral fractures of C6-7 and T2-3, and pneumonia.

\section{Social History}

The patient had been an active physician and lecturer until retirement 4 years before his death. Married with 2 adult children and 2 grandchildren, he enjoyed socializing and was an avid reader. From his early teens, he held many jobs with occupational toxin exposure: as a hydro worker at the shipyards, an army pharmacist who did compounding, and a dental assistant who worked with amalgam fillings. He travelled extensively throughout Europe and the Far East. His mother, who was of Ashkenazi Jewish descent, died at age 94 years of heart failure, and his father died after a myocardial infarction at age 40 years.

\section{Neuropathologic Examination}

Immunohistochemical and histochemical studies were performed on formalin-fixed paraffin-embedded brain sections, including the olfactory bulbs, prefrontal cortex, frontal cortex, amygdala, hippocampus, inferior temporal cortex, parietal cortex, occipital cortex, pons, substantia nigra, and cerebellum. All of these areas were immunopositive for $\alpha$-synuclein, and prominent labeling of neuronal Lewy bodies was apparent in the amygdala and hippocampus (Figure 1A and Figure 1B). Alzheimer pathology also was found consisting of intraneuronal phosphotau immunolabeling (Figure 1C, amygdala; Figure 1D, olfactory bulb) and $\beta$-amyloid 1-42 extracellular plaque immunolabeling in multiple regions (Figure 1E, amygdala; Figure 1F, inferior temporal lobe). Dual immunolabeling for $\beta$-amyloid 1-42 and Chlamydia pneumoniae, an intracellular bacterium previously observed in $\mathrm{AD}$ brains, ${ }^{9-11}$ revealed co-deposition in the amygdala and frontal cortex (Figure 1G and Figure 1H).

Numerous bodies consistent with corpora amylacea were observed after examining the olfactory bulbs, optic tract and chiasm, amygdala, and hippocampus. Periodic acid-Schiff (PAS) staining was performed to evaluate the composition of these bodies, as some studies ${ }^{12,13}$ have shown 1,4 $\alpha$-glucan branching enzyme 1 gene mutations leading to polyglucosan body formation, often considered to be corpora amylacea, in the 

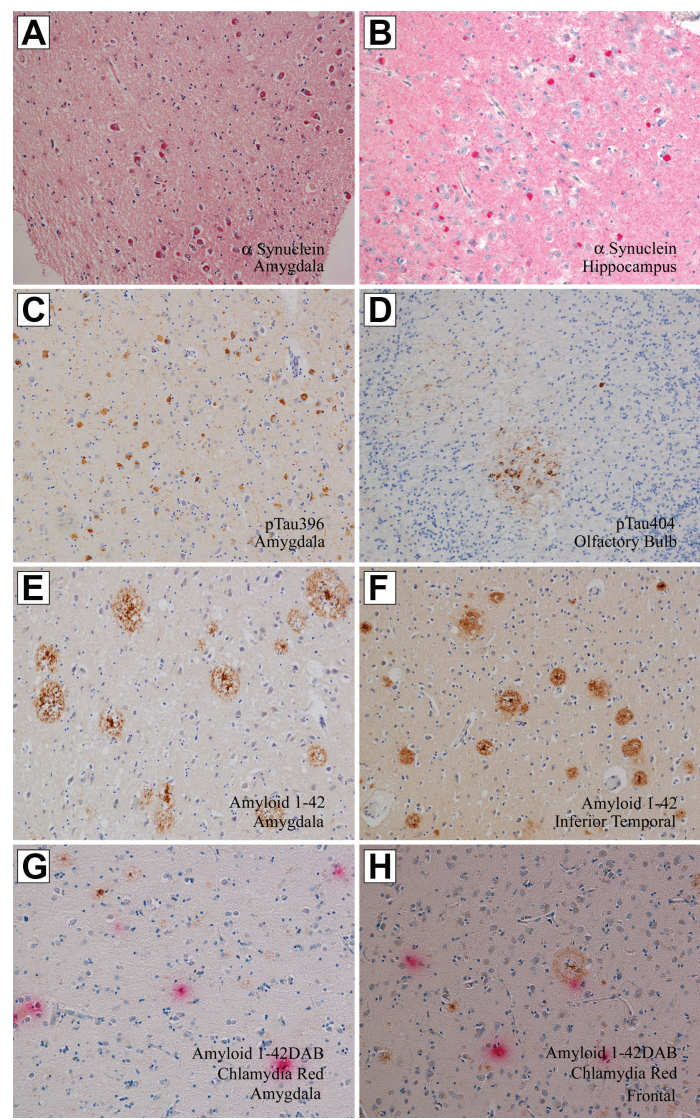

Figure 1.

Immunohistochemistry highlights mixed pathologic features in numerous brain regions. (A and B) Prominent $\alpha$-synuclein immunoreactivity (alkaline phosphatase red-new magenta; original magnification $\times 10$ ) reveals Lewy bodies in the amygdala and hippocampus; (C-F) immunolabeling (horseradish peroxidase/ 3,3'-diaminobenzidine, brown; original magnification $\times 10$ ) of phosphotau in neurofibrillary tangles (C, amygdala; $D$, olfactory bulb) and $\beta$-amyloid 1-42 (horseradish peroxidase/DAB, brown; original magnification $\times 10)$ in extracellular plaques ( $E$, amygdala; $F$, inferior temporal lobe) reveals Alzheimer disease characteristics; ( $\mathrm{G}$ and $\mathrm{H}$ ) dual immunolabeling for $\beta$-amyloid $1-42$ (horseradish peroxidase/DAB, brown; original magnification $\times 10$ ) and Chlamydia pneumoniae (alkaline phosphatase, red; original magnification $\times 10$ ) reveals pathologic changes in association with infection in both the amygdala and frontal cortex.

Ashkenazi Jewish population. A multitude of PAS-positive bodies were observed in the amygdala, olfactory bulbs, and the hippocampus (Figure 2). A decrease in the intensity and extent of PAS staining was
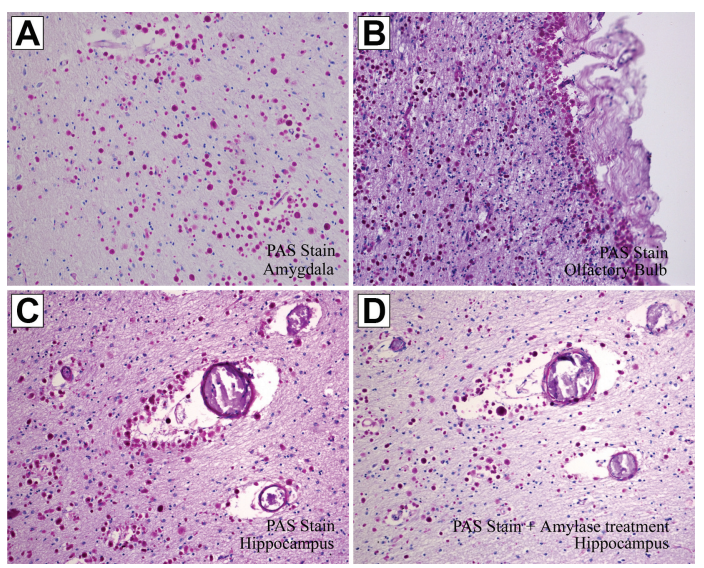

Figure 2.

Periodic acid-shift (PAS) staining of corpora amylacea/ polyglucosan bodies. (A-D) PAS-positive bodies (reddish-purple; original magnification $\times 10$ ) are apparent in the olfactory bulbs, amygdala, and the hippocampus; (D) amylase pretreatment on sections of the hippocampus followed by PAS staining results in a diminishment but not total loss of staining (original magnification $\times 10$ ).

observed after using amylase pretreatment on hippocampal sections followed by PAS staining (Figure 2D).

\section{Discussion}

The diagnosis of DLB in this case was based on the clinical history and symptoms, as well as the pathologic findings within multiple brain regions. However, the contributions of each of these findings to the initial symptoms and changes over time are more difficult to define given the varied and mixed pathologic features, including $\alpha$-synuclein accumulation, $\beta$-amyloid plaques, tau tangles, C pneumoniae labeling, and PAS-positive polyglucosan bodies. The features represent later Braak stages than would be expected based simply on a diagnosis of DLB. ${ }^{14}$ Braak staging delineates the extent of Parkinson and Alzheimer disease characteristics in different brain regions. Work by others ${ }^{15,16}$ has determined that in community settings, the cognitive deficit in DLB is most often associated with mixed structural and functional deviations, including vascular disease, $\mathrm{AD}$, and $\mathrm{DLB}^{15}$; Lewy bodies, $\beta$-amyloid plaques, and tau tangles are frequently observed in postmortem DLB brains. ${ }^{16}$ 
One surprising finding was immunolabeling for the intracellular, respiratory pathogen $C$ pneumoniae in the olfactory bulb, amygdala, and hippocampal sections of the brain, that had previously only been seen in AD cases. ${ }^{9-11}$ Dual immunolabeling for amyloid and $C$ pneumoniae is comparable to what we observed in AD brains. In DLB, AD neuropathology is often recognized. ${ }^{16}$ As the current case shows mixed pathologic features, the relationship of this dual immunolabeling may be explained. Further, DLB and AD have been shown to have comparable neuroinflammation. ${ }^{17}$ Thus, in this DLB case, the presence of $C$ pneumoniae infection, a trigger for neuroinflammation, in conjunction with the neuropathology of $\beta$-amyloid 1-42 plaques, could further support these associations.

Another surprising finding was the large spherical structures throughout the brain that stained prominently with PAS. They could be corpora amylacea or polyglucosan bodies. Pretreatment with amylase reduced some of the PAS staining, although this was not eliminated completely. As this individual was from Ashkenazi Jewish descent, adult polyglucosan body disease could be a further confounder for the clinical presentation. ${ }^{18}$ Polyglucosan body disease can be autosomal recessive or occur sporadically, and in both occurrences there is accumulation of abnormally branched glycogen due to dysfunction of the 1,4 alpha-glucan branching enzyme 1 gene. $^{19,20}$

Individuals with adult polyglucosan body disease typically present with symptoms in a range from 40 to 60 years and can experience frontotemporal dementia, motor neuron dysfunction, and neurogenic bladder. ${ }^{20}$ In comparison, individuals with a diagnosis of DLB are typically aged 70 to 85 years and present with symptoms of hallucinations, attention deficit, and late motor abnormalities. ${ }^{4}$

\section{Conclusion}

The rapid progression of this patient's illness was likely attributable to mixed pathologic features (DLB, AD, and polyglucosan body disease). The patient had characteristic clinical features to support the diagnosis of DLB, such as visual hallucinations and fluctuating cognition; however, analyses using single-photon emission computed tomography and positron emission tomography scans could have helped in pinpointing the disease processes. The patient's medical and social history were informative: he was a lifelong smoker with hypertension and coronary artery disease, he had a history of multiple physical traumas, and he had Ashkenazi Jewish heritage. All of these elements may have contributed to the neuropathologic changes we described. Follow-up investigation of this case will include genetic testing of tissues for $G B E$ mutations and real-time quantitative reverse transcription-polymerase chain reaction for molecular evaluation of infection with $C$ pneumoniae in brain tissues.

\section{Acknowledgments}

We thank the family and family caregivers for sharing their story. In addition, we thank Greg McDonald, DO, and Denah Appelt, $\mathrm{PhD}$, for their insightful discussions, and Ms Gwendolyn Harley for tissue sectioning. Last, we thank the Stanley E. Essl, DO '75 Endowed Memorial Research Fund for Neurodegenerative Diseases and the Center for Chronic Disorders of Aging for supporting this work.

\section{References}

1. American Psychiatric Association. Diagnostic and Statistical Manual of Mental Disorders. 5th ed. Washington, DC: American Psychiatric Publishing Inc; 2013.

2. Vann Jones SA, O'Brien JT. The prevalence and incidence of dementia with Lewy bodies: a systematic review of population and clinical studies. Psychol Med. 2014;44(4):673-683. doi:10.1017/ S0033291713000494

3. Galasko D. Lewy body disorders. Neurol Clin. 2017;35(2):325-338. doi:10.1016/j.ncl.2017.01.004

4. McKeith IG, Boeve BF, Dickson DW, et al. Diagnosis and management of dementia with Lewy bodies: fourth consensus report of the DLB consortium. Neurology. 2017;89(1):88-100. doi:10.1212/ WNL.0000000000004058

5. Gelb DJ, Oliver E, Gilman S. Diagnostic criteria for Parkinson disease. Arch Neurol. 1999;56(1):33-39.

6. Donaghy PC, McKeith IG. The clinical characteristics of dementia with Lewy bodies and a consideration of prodromal diagnosis. Alzheimers Res Ther. 2014;6(4):46. doi:10.1186/alzrt274

7. Kovacs GG, Alafuzoff I, Al-Sarraj S, et al. Mixed brain pathologies in dementia: the BrainNet Europe consortium experience. Dement Geriatr Cogn Disord. 2008;26(4):343-350. doi:10.1159/000161560 
8. Aarsland D, Ballard CG, Halliday G. Are Parkinson's disease with dementia and dementia with Lewy bodies the same entity? J Geriatr Psychiatry Neurol. 2004;17(3):137-145. doi:10.1177/ 0891988704267470

9. Balin BJ, Gérard HC, Arking EJ, et al. Identification and localization of Chlamydia pneumoniae in the Alzheimer's brain. Med Microbiol Immunol. 1998;187(1):23-42.

10. Hammond CJ, Hallock LR, Howanski RJ, Appelt DM, Little CS, Balin BJ. Immunohistological detection of Chlamydia pneumoniae in the Alzheimer's disease brain. BMC Neurosci. 2010;11:121. doi:10.1186/1471-2202-11-121

11. Gérard HC, Dreses-Werringloer U, Wildt KS, et al. Chlamydophila (Chlamydia) pneumoniae in the Alzheimer's brain. FEMS Immunol Med Microbiol. 2006;48(3):355-366.

12. Hussain A, Armistead J, Cushulak L, et al. The adult polyglucosan body disease mutation GBE1 c.1076A $>C$ occurs at high frequency in persons of Ashkenazi Jewish background. Biochem Biophys Res Commun. 2012;426(2):286-288. doi:10.1016/j.bbrc.2012.08.089

13. Lossos A, Meiner Z, Barash V, et al. Adult polyglucosan body disease in Ashkenazi Jewish patients carrying the Tyr329Ser mutation in the glycogen-branching enzyme gene. Ann Neurol. 1998;44(6):867-72. Pdoi:10.1111/j.1574-695X.2006.00154.x

14. Uchikado H, Lin WL, DeLucia MW, Dickson DW. Alzheimer disease with amygdala lewy bodies: a distinct form of alpha-synucleinopathy.
J Neuropathol Exp Neurol. 2006;65(7):685-697. doi:10.1097/ 01.jnen.0000225908.90052.07

15. Schneider JA, Arvanitakis Z, Bang W, Bennett DA. Mixed brain pathologies account for most dementia cases in community-dwelling older persons. Neurology. 2007;69(24):2197-2204. doi:10.1212/ 01.wnl.0000271090.28148.24

16. Ballard C, Ziabreva I, Perry R, et al. Differences in neuropathologic characteristics across the Lewy body dementia spectrum. Neurology. 2006;67(11):1931-1934. doi:10.1212/01.wnl.0000249130.63615.cc

17. Mrak RE, Griffin WS. Common inflammatory mechanisms in Lewy body disease and Alzheimer disease. J Neuropathol Exp Neurol. 2007;66(8):683-686. doi:10.1097/nen.0b013e31812503e1

18. Bit-Ivan EN, Lee $\mathrm{KH}$, Gitelman D, et al. Adult polyglucosan body disease with GBE1 haploinsufficiency and concomitant frontotemporal lobar degeneration. Neuropathol Appl Neurobiol. 2014;40(6):778-782. doi:10.1111/nan. 12144

19. Bigio EH, Weiner MF, Bonte FJ, White CL. Familial dementia due to adult polyglucosan body disease. Clin Neuropathol. 1997;16 (4):227-234.

20. Boulan-Predseil P, Vital A, Brochet B, Darriet D, Henry P, Vital C. Dementia of frontal lobe type due to adult polyglucosan body disease J Neurol. 1995;242(8):512-516.

(c) 2019 American Osteopathic Association 